\title{
Contact-centers and Artificial Intelligence
}

\section{"Intellectual Archive" magazine interviews the Voice Networks expert of Rostelecom Digital Technologies Company, Moscow}

Ruslan Makrushin talks to "Intellectual Archive" (I.A.) about trends in the advanced contact centers market, the future of their architecture and the application of the artificial intelligence.

Background:

- Participation in creation of one of the largest contact centers in Russia - the Nationwide Call Center of "Sberbank of Russia" PJSC (2012),

- Migration of the corporate contact center of Orange Business Services Company from one city to another with no service interruption (2017),

- Participation in development of Rostelecom Digital Technologies, one of the world's largest telephony and video conferencing system for the public sector on a nationwide scale (20192021),

- Teaching as a facilitator,

- Acting expert in the telecommunication organization International Avaya Users Group (USA):

https://iaug.informz.net/informzdataservice/onlineversion/ind/bWFpbGluZ2luc3RhbmNlaWQ 9MzM5NDk4NCZzdWJzY3JpYmVyaWQ9MzgzMjg0MDU0

\section{- I.A.: Could you announce the market requirements for contact centers in the modern world?}

- That is a tough question, yet now we can say that factors, which started to come out yesterday are becoming relevant today and will be decisive tomorrow. Here we can distinguish two areas: technological and administrative.

Speaking of the technological area, which is closer to me, serving customers through all modern access channels, such as web chat, social network, email, SMS, and not just through the traditional voice channel, becomes more crucial. It is true that voice channels are still quite common now. Nevertheless, those companies in particular, able to consider the client's request to the contact center from the perspective of meeting the needs of the client in general, without reference to the channel of application, are sure to succeed. Consideration and evaluation of the client's request, so to speak, in the range of one particular channel, for example, voice or web chat, should be a thing of the past. 


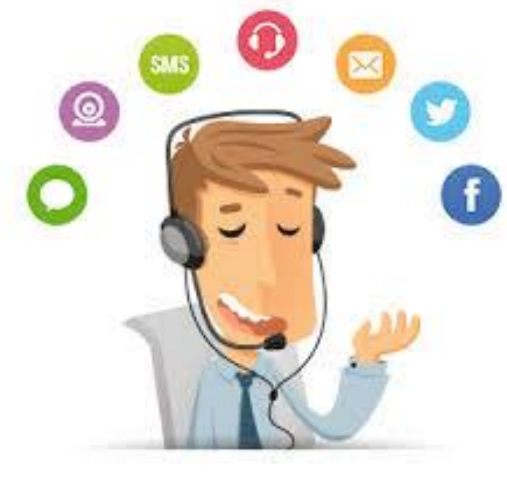

\section{- I.A.: Will it require new skills from the contact center operators?}

- This will require new technological skills from operators specifically. Experience with the issues advised change only in compliance with changes in the field the operator specializes in. Nevertheless, the operator will have to master new tools when interacting with the client. Regardless of how the client contacted the contact center - by e-mail, via Internet chat, from a social network, or simply by phone, the operator will have the whole view of interaction with this client and all his requests. And the operator needs to be able to solve the client's requests using all variety of tools provided to him, and, of course, by means of all access channels used for the operator - client interaction. We will face the transition from one model to another. In short, this is the transition from the "device" model to the "operator-client" model during the complete working cycle.

\section{- I.A.: What does the client need it for? Is not it too complex for him?}

- The client just uses a convenient way of contact. In general, currently we have plenty of opportunities to make an order. No matter how we contact the contact center and no matter how we interact later, a good share of work lies with the operator and should shift to applications based on artificial intelligence. Both the operator and the applications see the whole story and can tell, for example, what the client has to do next, and the hint will reach the client via the channel of convenience for him, for example by SMS.

\section{- I.A.: What extent of efficiency the artificial intelligence may have in customer service sector?}

Artificial intelligence (AI) is changing the technological infrastructure. Everything we talk about, whether chatbots or multichannelling, can be based on artificial intelligence. Generally, AI applications, operating with typical question-and-answer, reduce the load on operators. Chatbots process their scripts; thus, can involve other applications and one or another operator. There are also so-called cognitive computations. This is a simulation of human thinking, which facilitates identification of behavioral patterns and analyzes data. Then the AI application interacts with clients in such a way that they are practically indistinguishable from a live 
operator. Altogether, according to research agencies, the role of AI is cost saving of businesses in the field of customer service one hundred fold!

\section{- I.A.: What will the customer impressions be based on, which are of that much importance for business?}

- Customer impressions in general will be based on the same criteria. Psychology of people does not change as dramatically as technology. It is important to evaluate these very criteria differently, keeping up with the times, namely, using the multichannelling there again. There is a relevant term for this - the "Omni channel". This is the one customer-oriented approach we heard so much about. In order to make impressions of the client addressing the contact center positive, among other challenges, we need to provide the client with the opportunity to switch between access channels. The mechanism to implement the possibility of such changeovers should be extremely clear to the client and cause no feeling of confusion. This is the main thing.

\section{- I.A.: Which of the currently known contact center parameters will be the most important?}

- Today we operate with the following so-called metrics: Average Handling Time (AHT) average call service time, Customer Satisfaction (CSAT) - customer satisfaction, First Call Resolution (FCR) - an index of the request solution at the first call. Contact centers are probably to abandon these indicators gradually. It is clear from their names already that operators may doubt them. We need indicators that would inspire operators interact efficiently with the client. It is possible to develop elements in the course of interaction with each specific client, that the client is satisfied and not satisfied with. Communication style, language, and possibly the operator himself can be selected using automated voice analysis during the initial client request.

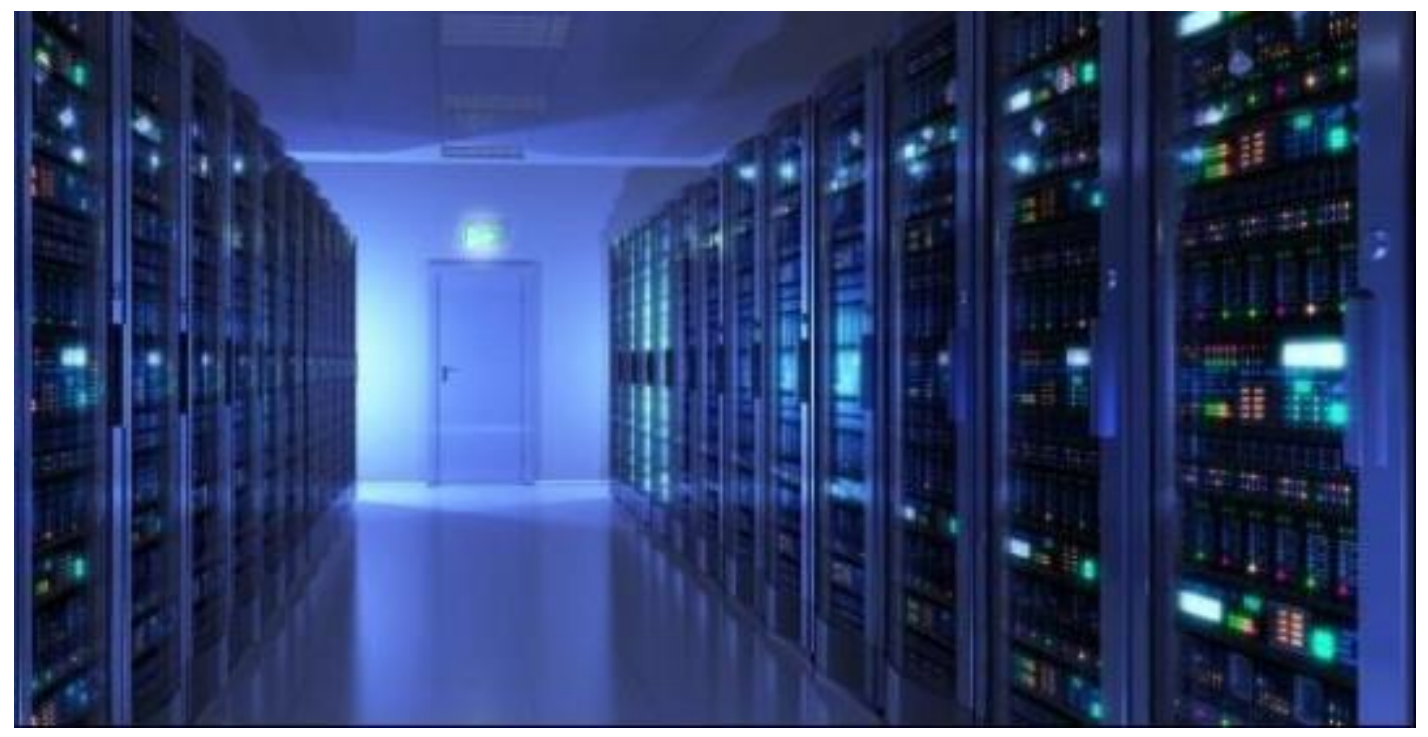

\section{- I.A.: Will there be something making it possible to reduce service price?}


- Self-service systems (IVR) have always been perceived as an opportunity to reduce the number of contact center operators and, thus, reduce the contact center maintenance cost. They will still be necessary components, but mainly to facilitate completion of own tasks by the client. Basically, the client will contact by phone just when he did not manage to solve the issues in the self-service system. Of course, traditional telephone communication will continue to support all other channels of access to the contact center.

\section{- I.A.: Thus, will human operators stay with the play?}

- They will. It is not least because of the future that will not make us away from complex, nonstandard customer requests. Another thing is that special operators - bots are gaining more and more intelligence. For one thing, modern software makes it possible to start interacting with the client. Bots process an increasing number of requests, conducting longer and longer interactions. At the same time, speech recognition technologies are improving. Thus, bots will be fully functional in interaction with us, humans, while having strict logic, a significant knowledge base and endless patience. Human operators will join interaction with the client at the right time and even in the right place.

\section{- I.A.: Did you say in the right place?}

- Yes, exactly in the right place they need. Technologies and capabilities will easily allow and make it feasible for operators to work remotely now, in the places with access to the Internet. It is not always necessary for operators to work in a crowded noisy office. This may be required at the initial stage of the operator's work during his training. Both training and, testing of operators for sure, can be online. Thus, the digital CV of the operator, with the operator's skills listed, will be simply adjusted subsequently by monitoring algorithms and by clients themselves, who are invited to evaluate the operator's work.

\section{- I.A.: Can the client interaction through the contact center bring some benefit to the company in the future?}

- It can and it will be of use. However, it is important to work in the interests of the client in every aspect, to take care of his satisfaction. An aggressive service with sending offers to the client's devices is not acceptable. If however the client expresses interest in some kind of information, for example, the campaigns held by the company, it is registered in the database. Generally, analysis of client interaction and interaction with a person who has just addressed the center can have plenty of useful information. This is both identification of possible sales and trends in the future.

\section{- I.A.: You highlighted the administrative sphere, speaking about the development of contact centers. What do you mean by this?}


- The technological component is primary. Its development decides on evolution of related aspects of business. There are new requirements for employees of the contact centers, operators, group leaders, supervisors. Here we deal with the administrative sphere.

\section{- I.A.: Who are supervisors?}

- These are employees involved in evaluating the personnel performance, in staff management and staff development. These are usually the operators in the past. The tools for the supervisors' activities are a variety of software, which is usually regular, but it is not always the case. Thirdparty software products can also be used, for example, programs developed "for the client". In this case, we are talking about the so-called computer integration. In general, if we talk about the future, the number of supervisors required for business will reduce sharply. Automated realtime monitoring systems will do their job. These same systems, analyzing words, tone of voice, emotions, will be able to make a decision on involving a supervisor.

\section{- I.A.: Finally, can you tell us about the technical complexities that an engineer serving a contact center may encounter?}

- For me personally, the word "difficult" means interesting. A project on the migration of a contact center from one city to another comes to mind. This operation should be performed without interruption in service provision. We are talking specifically about physical relocation

of all servers, gateways, switches and other architecture nodes. The task is feasible in case the contact center nodes are duplicated. For example, the main telephony server is usually duplicated. In short, the load is transferred to a part of the nodes. Let us call them switching ones. Duplicates move, connect to the network to be ready to take the load. When ready, traffic is redirected to the new site. It is not all that simple. All configuration changes on switching nodes should be done on inactive ones as well, which are already on the new site. After all, these inactive ones become switching once they take the load. Most importantly, you need to be ready for the fact that a certain node after transportation will not operate as required. In general, such project is a chain of separate works, and once there is a problem at a certain stage, it is difficult to move on. Therefore, everything is clearly thought out and recorded, a rollback plan is determined. By the way, cloud solutions are of help to simplify the task. Migrating a server "from cloud to cloud" is much easier. Besides, speaking of the future, businesses will use purely virtual services, that is those of the cloud contact centers, which are offered by integrator companies even as we speak.

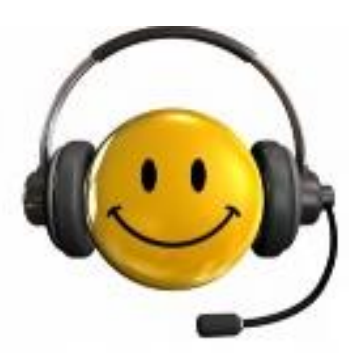

